\title{
Surgical Treatment of Epilepsy
}

\section{Epilepsi'nin Cerrahi Tedavisi}

\section{Davut Ceylan' ${ }^{1}$ Can Yaldız ${ }^{2}$}

${ }^{1}$ Sakarya University Medical Faculty, Department of Neurosurgery

${ }^{2}$ Sakarya University Education and Research Hospital, Department of Neurosurgery

\begin{abstract}
The purpose in epilepsy treatment is the complete control of seizures and the minimization of the side effects of antiepileptic drugs. Epilepsy clinics deal a wide range of diagnostic and therapeutic problems of patients with uncontrolled seizures. The choice of surgical treatment must be considered in such patients who are resistant medical treatment.
\end{abstract}

Keywords: Epilepsy, surgery

Aplication: 15.05.2013 Accepted: 08.07.2013

\section{Introducion}

Epilepsy is one of the most common neurological disorders with a prevalence of 5 to 9 in 1000 people'. The purpose in epilepsy treatment is the complete control of seizures and the minimization of the side effects of antiepileptic drugs (AEDs) ${ }^{2}$. Although the seizures can be controlled by antiepileptic treatment in almost $65 \%$ of epilepsy patients, they cannot be controlled in 35\% of patients despite maximal medical regimen ${ }^{1,3}$. The choice of surgical treatment must be considered in such patients who are resistant medical treatment.

\section{Resistance to Medical Treatment}

No consensus exists on the definition of treatment-resistant epilepsy in the literature. There are significant differences between the treatment method used in various studies, number and duration of drugs, and the frequency and exposure times of seizures ${ }^{4}$. The approach assuming that patients who are candidates for epilepsy surgery are treatment-resistant and adopted by many authors now is based on the failure of two consecutive monotherapies and a successive combination therapy, $y^{5,6}$.

The purpose and patient selection in surgical intervention

About half of patients with resistant seizures are bound to undergo epilepsy surgeries ${ }^{1,3}$.

The purpose of epilepsy surgeries is to stop or reduce the frequency of seizures as much as possible, while creating no additional neurological deficits in the patient 
and improving the patient's quality of life ${ }^{3}$. There are two conditions involving an epilepsy surgery. The first is the presence of an epilepsy resistant to drug therapy, and the second is the presence of an epileptic origin curable by surgery in the patient. Surgery should be comfortably recommended to patients who are likely to have a higher chance of recovery. Palliative treatment should be recommended even to patients with low chance of full recovery in order to reduce the frequency of seizures ${ }^{7,8}$. Etiological studies of epilepsy patients can reveal many of the underlying factors. These factors may be congenital or acquired. In such cases, epilepsy can be treated with surgical intervention (Table 1,2)

Table 1 : Epilepsies curable by surgery (From Ref.3)

\begin{tabular}{l}
\hline A. Epilepsies with focal pathologies and likely to receive lim- \\
ited resection: \\
-Mesial temporal lobe epilepsy \\
-Malformations of cortical development \\
-Benign/low-grade tumors \\
-Vascular anomalies \\
-Post-infectious changes \\
-Post-traumatic changes \\
-Partial epilepsies with normal MRI (MR negative epilepsies) \\
\hline B. Diffuse epilepsies generally requiring multilobular resection \\
or hemispherectomy: \\
-Widespread cortical developmental malformation (i.e. Hemimega- \\
lensephaly) \\
-Other developmental deficiencies of the brain (Sturge Weber \\
Syndrome) \\
-Widespread pre- and postnatal vascular lesions \\
-Hemiconvulsion-Hemiphlegia-Epilepsy Syndrome \\
-Rasmussen encephalitis \\
\hline C. Epilepsies relieved by disconnection operation: \\
-Lennox-Gastaut \\
-Falling seizures during the syndromes \\
-Landau Kleffner Syndrome
\end{tabular}

Table 2 : Epilepsies secondary to lesions (From Ref.3)

A. Malformations of cortical developmental (MCDs)

B. Tumors: Low-grade gliomas (Astrocytoma, oligodendroglioma, ganglioglioma, mixed glioma, pleomorphic xanthoastrocytoma),

meningioma, dysembryoplastic neuroepithelioma

C. Vascular malformations: Arteriovenouss malformations, cavernoma

D. Cystic lesions: Porencephalic cysts

E. Infectious and post-infectious changes, tuberculoma, neurocysticercosis

F. Vascular lesions

G. Post-traumatic lesions

\section{Preoperative patient evaluation}

The aim of epilepsy surgical interventions is the surgical excision of the epileptogenic tissue. No neurological deficits should be induced by the operation. Therefore, it is important to localize the epileptogenic tissue as well as important structures in the brain including the motor, speech and memory areas in the brain A combination of different methods is used to localize the epileptogenic zone. Methods used to preoperatively evaluate the patients are invasive and non-invasive methods ${ }^{3}$. (Table 3) Long-term video-EEG(electroencephalogram) monitoring is at the center of preoperative evaluations. EEG is vital in confirming the diagnosis of epilepsy, classifying seizure types and identifying the epileptogenic focus. Video-EEG plays an important role in deciding for preoperative diagnosis and surgical method thanks to the improvements in examination techniques and imaging analysis methods $3,9,10$.

Table 3 : Stages of evaluation before epilepsy surgery (From Ref.3)

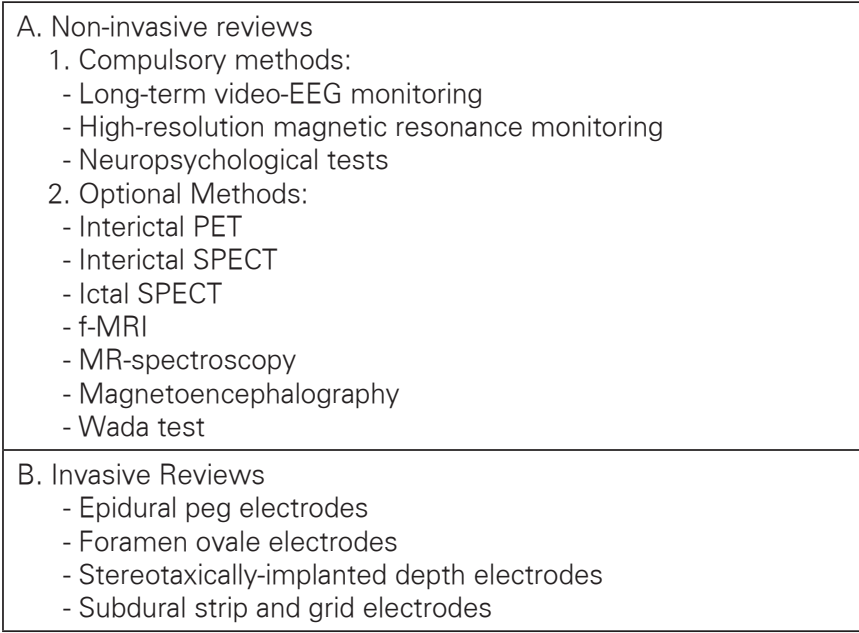

Magnetic resonance imaging (MRI) has a central role in neuroradiological examination. Very important information has become available for preoperative diagnosis thanks to the improvements in examination techniques and imaging analysis methods ${ }^{3}$. Functional deficits induced by focal epilepsy can be measured by the changes in positron emission tomography (PET) and single-photon emission computed tomography (SPECT). PET is $60-90 \%$ sensitive in temporal lobe epilepsies, $60 \%$ sen- 
sitive where there are no findings in $\mathrm{MRI}$, and almost $100 \%$ in patients with hippocampal atrophy ${ }^{11,12}$. Functional MRI and Wada tests are used in speech lateralization and these two diagnostic methods are highly correlated. The correlation is highest, 91-100\%, in left hemispheric speech representation, and $67-100 \%$ in the right hemisphere ${ }^{3}$.

Invasive examinations where intracranial electrodes are placed are used when localization cannot be achieved by noninvasive examinations. They include Peg electrodes, foramen ovale electrodes, stereotaxically-implanted depth electrodes and subdural strip and grid electrodes. Neuropsychological evaluation forms an important step of the preparatory process for epilepsy surgery. Cerebral lesion is localized by imaging methods and electrophysiological examinations, but the effects of this lesion on cognitive functions and behavior remain to be uncertain. Neuropsychological tests reveal how cognitive functions and behaviors are affected following brain damage. By doing this, they help to localize the areas where cognitive functions are preserved or impaired ${ }^{13}$.

\section{Surgical treatment}

After completion of the preoperative period, choice of the surgical method will be decided according to the localization of the epileptiform region and the type of epileptic seizure (Table 4). Interventions aimed at temporal lobe epilepsy and particularly at mesial temporal lobe epilepsies are the major portions of the epilepsy surgery. In situations where the epileptogenic tissues are closely associated with functionally important brain regions, large resections should be avoided, as they will result in significant neuropsychological losses of function ${ }^{14}$. So, following interventions called En-bloc resection where temporal lobes are entirely removed, new operational techniques are developed thanks to the improvements in microsurgical techniques, which ensured the protection of lateral temporal lobe structures and the resection could be limited to the temporal pole and mesial temporal structures (antero-mesial resection) or only to mesial structures (selective amygdalohippocampectomy). Neuropsychological deficits and particularly the losses in memory are minimized with these selective operational techniques ${ }^{3}$. The operative strategy in the treatment of extratemporal epilepsies is aimed at the detected structural lesion. Where the epileptogenic tissues are very close to the functionally important brain regions, significant losses of function may occur due to wide resection. In this case, subpial transection technique, an alternative method, is recommended ${ }^{14}$. This method allows the separation of intercortical horizontal bands at a distance of $5 \mathrm{~mm}$, also protecting the vertical structures and the pia. This prevents the horizontal spread epileptic activity, also protecting the functions organized by vertical cellular links.

Table 4 : Current methods used in epilepsy surgery are listed below

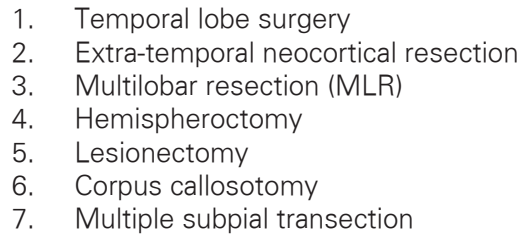

Common pathologies (hemimegalencephaly, other diffuse cortical dysplasias, Sturge Weber syndrome, Rasmussen's encephalitis) are typically catastrophic processes that result in severe epilepsies in childhood and are very difficult to treat, and their surgical treatments are generally based on diffuse multi lobular resection or hemispherectomies ${ }^{3}$. Corpus callosotomy is among other forms of intervention. It is indicated with the treatment of seizures such as falling seen in generalized epilepsies including the Lennox-Gastaut syndrome. In this procedure, the $2 / 3$ portion of anterior corpus callosum is resected, and the communication between both frontal and parietal lobes is interrupted ${ }^{15}$.

Engel's classification has been identified as the most commonly used classification system for the evaluation of seizure control postoperatively ${ }^{16}$. (Table 5) Correlation will be achieved between postoperative success and other clinical findings thanks to this classification system. Epilepsy surgery is rarely associated with complications. Mortality is about $0,5 \%$ in temporal lobe resections, $0,8 \%$ in extratemporal lobe resections and $2 \%$ in hemispherectomies. Neurological complications are less 
than $5 \%$, with a majority of them fully recovering within several weeks. Temporal resections may result in anop$\operatorname{sias} 13$.

Table 5 : Classification of post-operative seizure control based on Engel's classification

\begin{tabular}{|l|}
\hline Class I: Free of disabling seizures \\
1A: Totally overcoming seizures \\
1B: nondisabling simple partial seizures only since surgery \\
1C: some disabling seizures after surgery, bur free of disabling \\
seizures for at least 2 years \\
1D: Generalized seizures due to interruption of antiepileptic \\
drugs \\
\hline Class II: Rare disabling seizures ("almost seizure free") \\
2A: initially free of disabling seizures but has rare seizures now \\
2B: rare disabling seizures since surgery \\
2C: more than rare disabling seizures since surgery, but rare \\
seizures for the last 2 years \\
2D: nocturnal seizures only \\
\hline Class III: Worthwile improvement \\
3A: worthwile seizure reduction \\
3B: prolonged seizure-free intervals amounting to greater than \\
half the followed-up period, but not < 2 years \\
\hline
\end{tabular}

\section{Kaynaklar}

1. Spencer DD, Spencer SS, Mattson RH, Williamson PD, Novelly RA. Access to the posterior medial temporal lobe structures in the surgical treatment of temporal lobe epilepsy. Neurosurgery 1984;15(5):667-71.

2. Recommended guidelines for diagnosis and treatment in specialized epilepsy centers. Epilepsia 1990;31(Suppl):1-12.

3. Baumgartne $C$, Watschınger SA, Czech T, Eder HG, Feichtinger M, Feucht M,Gallmetzer P et all. Cerrahisi Öncesi Değerlendirme Ve Epilepsi Cerrahisi: Güncelleme. Epilepsi 2008;14(2): 111-130

4. Berg AT. Defining intractable epilepsy. Adv Neurol 2006; 97: 5.10.

5. French JA. Refractory epilepsy: one size does not fit all. Epilepsy Curr 2006; 6: 177.80

6. Aicardi J, Shorvon SD. Intractable epilepsy. In: Engel J Jr, Pedley TA (eds). Epilepsy: A Comprehensive Textbook. Lippincott- Raven Publishers, Philadelphia, 1997; 1325.31.

7. Gilliam F. Optimizing health outcomes in active epilepsy. Neurology 2002; 58: S9.S20.

8. Gilliam FG, Fessler AJ, Baker G, et al. Systematic screening allows reduction of adverse antiepileptic drug effects: a randomized trial. Neurology 2004; 62: 23.7.

9. Engel J Jr, Wiebe S, French J, et al. Practice parameter: temporal

\section{Conclusion}

Patients who could have been treated in the early period are scheduled for a later date because surgical treatment is considered as the last choice of treatment in epilepsy. The decision of an early surgery for follow-up patients is taken where response to medical treatment is below the expected level, and there is an increase in cognitive loss or in pathological changes in $\left.M R\right|^{3,17,18}$. It has been demonstrated that neuropsychological and psychosocial results improve, the adverse effects of antiepileptic drugs are reduced when applied in high doses for an extended period, and severe epilepsies in the childhood have fewer negative effects on psychomotor improvement when early surgical intervention is chosen as the treatment option. A successful surgery ensures a significant decrease in mortality. A proper surgery is also an economically effective choice of treatment.

lobe and localized neocortical resections for epilepsy: report of the Quality Standards Subcommittee of the American Academy of Neurology, in association with the American Epilepsy Society and the American Association of Neurological Surgeons. Neurology 2003; 60: 538.47.

10. Bilir E. Adult Epilepsy Surgery : Presurgical Evaluations. Turk Nöroşirurji Dergisi 12: 7 - 15, 2002

11. Gaillard WD. Metabolic and functional neuroimaging. In: Wyllie E (ed). The Treatment of Epilepsy: Principles and Practice. 3rd ed.Lippincott Williams \& Wilkins, Philadelphia, 2001; 1053.66.

12. Ryvlin $P$, Bouvard $S$, Le Bars $D$, et al. Clinical utility of flumazenilPET versus [18F] fluorodeoxyglucose-PET and MRI in refractory partial epilepsy. A prospective study in 100 patients. Brain 1998; 121: 2067.81

13. Chelune GJ:The role of neuropsychological evaluation of the epilepsy surgery candidate, Wyler AR, Heumann BP (eds), The Surgical Treatment of Epilepsy, first edition, New York: Butterworth Heinemann 1994; 78-89

14. Morrell F, Whistler WW, Bleck TP. Multiple subpial transection: a new approach to the surgical treatment of focal epilepsy. J Neurosurg 1989; 70: 231.9. 
15. Roberts DW, Rayport M, Maxwell RE, et al. Corpus callosotomy In: Engel J Jr (ed). Surgical Treatment of the Epilepsies. 2nd ed.Raven Press, New York, 1993; 519.26.

16. Wieser HG, Blume WT, Fish D, et al. ILAE Commission Report. Proposal for a new classification of outcome with respect to epileptic seizures following epilepsy surgery. Epilepsia 2001; 42:282.6.
17. Helmstaedter C, Kurthen M, Lux S, et al. Chronic epilepsy and cognition: a longitudinal study in temporal lobe epilepsy. Ann Neurol 2003; 54: 425.32

18. Jokeit $H$, Ebner $A$. Long term effects of refractory temporal lobe epilepsy on cognitive abilities: a cross sectional study. J Neurol Neurosurg Psychiatry 1999; 67: 44.50. 